\title{
Transport Behaviour of Electroactive Species in lonic Compounds: A Focus on Li Diffusion through Transition Metal Oxide in Current Flowing Condition
}

\author{
Sung-Jai Lee and Su-Il Pyun* \\ Department of Materials Science and Engineering, Korea Advanced Institute of Science and Technology, \\ 335 Gwahangno, Yuseong-gu, Daejeon 305-701, Republic of Korea \\ (Received February 1, 2009 : Accepted February 11, 2009)
}

\begin{abstract}
This article reviewed transport behaviours of electroactive species in ionic compounds, focusing on chemical diffusion of $\mathrm{Li}$ through the transition metal oxide in a current flowing condition. For this purpose, a distinction has been first briefly made between migration and diffusion with respect to current, driving force and charge of electroactive species considered. Then, the equations for chemical diffusion are derived theoretically in open-circuit and current flowing conditions. Finally, the experimental methods such as ac impedance spectroscopy and current (potential) transient techniques are described in details for characterising chemical diffusion. In addition, the role of the thermodynamic enhancement factor in chemical diffusion is discussed.
\end{abstract}

Keywords : Chemical diffusion, Ionic compound, Chemical diffusivity, Current flowing condition, Thermodynamic enhancement factor.

\section{Introduction}

Mass transport (migration and diffusion including convection) in an electrochemical system is one of the most important subjects in the fields of modern applied electrochemistry and electrochemical engineering. ${ }^{1-4)}$ Especially, the high performance of electrochemical devices such as batteries, supercapacitors and fuel cells is highly dependent upon the mass transport rate of the electroactive species. ${ }^{5,6)}$

Mass transfer, that is the movement of electroactive species from one location in solution or electrode to another, arises either from differences in electrical or chemical potential at the two locations or from movement of a volume element of solution. In general, mass transfer can be classified into the following three types ${ }^{4)}$ : (i) migration: movement of a charged body under the influence of an electric field (gradient of electrical potential); (ii) diffusion: movement of an electroactive species under the influence of a gradient of chemical potential (concentration gradient); and (iii) convection: stirring or hydrodynamic transport (natural

*E-mail:sipyun@kaist.ac.kr convection or forced convection).

A rigorous solution is generally not very easy when all forms of mass transport are in effect. Hence, electrochemical systems are frequently designed under the condition where one or more of the contributions to the overall mass transfer are negligibly small. In the case of mass transport through the electrolyte, for example, migration can be reduced to negligible levels by addition of a supporting electrolyte with a much larger concentration than that of the electroactive species, or convection can be avoided by preventing stirring and vibrations. On the other hand, in the case of mass transport through the electrode, convection is generally neglected due to the rigid transport media. Hence, only diffusion and migration fluxes dominantly contribute to the total mass transport.

In many electrochemical systems, mass transfer in the electrode is more important than that in the electrolyte, because the former is kinetically more sluggish than the latter, hence is the rate-determining step of the electrochemical process. ${ }^{7-10)}$ In the electrode, an electroactive species is transported by both migration and diffusion, and hence the total flux can be separated into diffusion and migration fluxes reflecting the diffusive and 
migrational components to the total flux of the electroactive species. To investigate mass transport in the electrode, it is necessary to identify quantitatively the relative contributions of migration and diffusion to the total transport.

First, let us consider a migration process. When the concentration gradients are very small, the total transport is carried mainly by migration. In this case, the driving force of migration is an electric field. Considering a linear electric field, the driving force is given as follows ${ }^{4)}$ :

$$
\frac{\partial \phi}{\partial x}=\frac{\vec{E}}{L}
$$

where $\phi[\mathrm{V}]$ means the electrical potential, $x[\mathrm{~cm}]$ the distance from the electrode/electrolyte interface, $\vec{E}[\mathrm{~V}]$ the electric field, and $L[\mathrm{~cm}]$ represents the electrode thickness. Under the electrical potential gradient, the flux by migration can be expressed by

$$
j_{i}=\frac{z_{i} F D_{k, i} c_{i}}{R T} \frac{\partial \phi}{\partial x}
$$

where $z_{i}[-]$ is the charge of the $i$ th electroactive species, $D_{k, i}\left[\mathrm{~cm}^{2} \mathrm{~s}^{-1}\right]$ the component diffusivity of the $i$ th species, and $c_{i}\left[\mathrm{~mol} \mathrm{~cm}^{-3}\right]$ means the concentration of the $i$ th species. The notations $F, R$, and $T$ are commonly used symbols. From Eq. (2), it is confirmed that a pure migration process can occur only when the charged species moves under the electrical potential gradient.

On the other hand, when the electrical potential gradient is not established within the electrode or uncharged electroactive species transport, the total transport is crucially controlled by diffusion. In this case, the flux by diffusion can be expressed by ${ }^{4}$

$$
j_{i}=D_{k, i} \frac{\partial c_{i}}{\partial x}
$$

From Eq. (3), it is found that a pure diffusion process may occur only when there is no electrical potential gradient within the electrode.

Here, it should be stressed that in real electrochemical systems, the transport mechanism of the electroactive species through the electrode is not fixed at the specific electrode system by itself, but it is rather simultaneously determined even at any electrode system by the driving force of the transport. For instance, let us consider oxygen transport through the stabilised zirconium dioxide. When a relative large current flows through the electrode, the potential gradient is established in the electrode, and the oxygen ion (or charged oxygen vacancy) transports purely via migration by the electrical potential gradient. ${ }^{11-19)}$ In contrast, in case that there is no current flow through the electrode, oxygen (or oxygen vacancy) transports in a neutral form via diffusion due to the electrochemical potential gradient. ${ }^{11,20-22)}$ Two different transport mechanisms are illustrated schematically in Fig. 1.

In the more specific case, let us consider a transport of the neutral electroactive species $i^{*}$, in which the transport of all species within the transport medium is involved, which is generally called chemical (bipolar) diffusion. ${ }^{5,6,23)}$ In this case, we assume that there is no external electrical field and the overall charge neutrality holds. Hence, the total flux density is expressed in terms of the individual kinetic and thermodynamic properties of electroactive species:

$$
\sum_{i} z_{i} j_{i}=0
$$

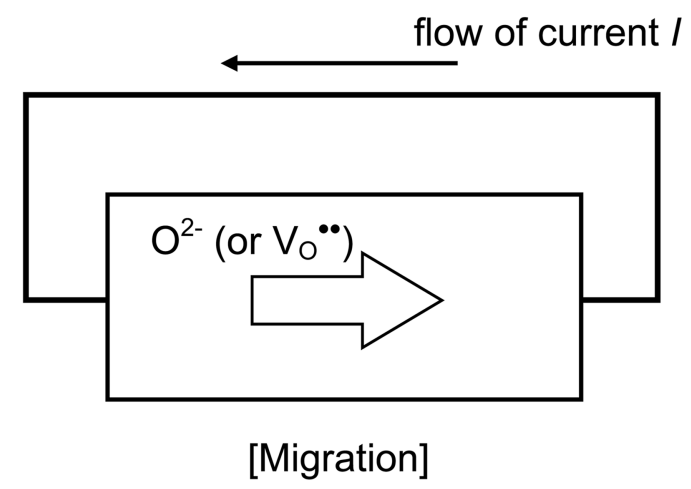

(a)

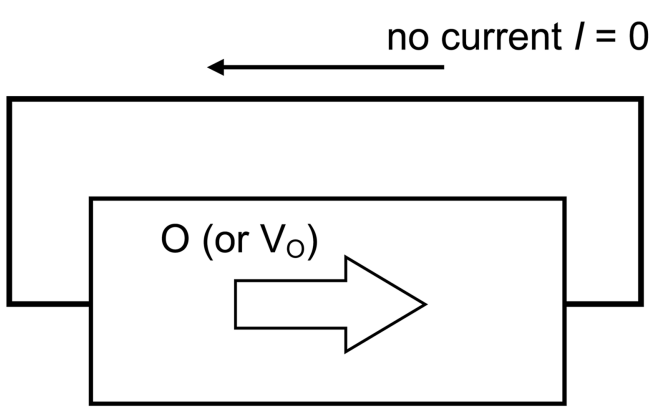

[Diffusion]

(b)

Fig. 1. Schematic diagrams of oxygen transport via (a) migration by the electric potential gradient and (b) diffusion by the electrochemical potential gradient. 
where $i$ represents each electroactive species.

Although the internal electrical field gradient $\nabla \phi$ is not known, it can be eliminated from Eq. (4). Then, the overall flux density equation of the neutral species $i^{*}$ for chemical diffusion is expressed in terms of transport quantities and activity gradients related to charged species $i$ and to all other species $j$.

$$
\begin{aligned}
j_{i^{*}} & =-D_{k, i}\left[\left(1-t_{i}\right) \frac{\partial \ln a_{i}}{\partial \ln c_{i *}}-\sum_{j \neq i} t_{j} \frac{z_{i}}{z_{j}} \frac{\partial \ln a_{j}}{\partial \ln c_{i *}}\right] \nabla c_{i} \\
& =-\tilde{D_{i}} \nabla c_{i}
\end{aligned}
$$

Here, $t_{i}[-]$ means the transference number of the $i$ th charged species. $\tilde{D}_{i *}\left[\mathrm{~cm}^{2} \mathrm{~s}^{-1}\right]$ is the chemical (apparent) diffusivity of the neutral form $i^{*}$ for the charged species $i$, for example, $i^{*}=\mathrm{Li}$ for $i=\mathrm{Li}^{+}$. This equation includes the influence of the motion of species $j$ other than species $i$ on the motion of electroactive species $i$ by the effect of the internal electrical field.

Eq. (5) containing the chemical diffusivity is usually adopted to analyse the transport of electroactive species within the transport medium in real electrochemical systems. It is noted that the component diffusivity $D_{k, i}$ is independent of the motion of any other species and is not influenced by the internal electrical field, whereas the chemical diffusivity $\tilde{D}_{i}$ * crucially depends upon the motion of any other species, as shown in Eq. (5). This indicates that the transport of electroactive species $i^{*}$ is enhanced or retarded by the motion of the other species induced by the internal electric field.

The consideration of all the electroactive species participating the transport usually fails due to the complexity of the situation and the difficulty in reasonable evaluation of the parameters needed for calculation. Fortunately, in many electrochemical systems of practical importance, the situation becomes simplified by the fact that two types of species dominate the transport phenomena. Recently, we have analysed theoretically chemical diffusion in an open-circuit condition, then discussed on the basis of the local equilibrium constraint. ${ }^{23)}$ This work is a further stage of the continuing reports on the collective programme of investigating the chemical diffusion through the ionic compounds.

Under this circumstance, this work covers the theoretical treatment of chemical diffusion of Li through the transition metal oxide containing only two electroactive species, i.e., Li ion and electron in a current flowing condition. Then, the equation for chemical diffusion derived under the current flowing condition is compared qualitatively with that derived under the open-circuit condition. Finally, the experimental methods are detailed to determine the chemical diffusivity and the thermodynamic enhancement factor.

\section{Theoretical approaches to chemical diffusion with aid of simulation experiments}

\subsection{Chemical diffusion in an open-circuit condition}

From the flux density equation of the $i$ th electroactive species, $j_{i}\left[\mathrm{~mol} \mathrm{~cm} \mathrm{~cm}^{-2} \mathrm{~s}^{-1}\right]$ with the assumption of onedimensional transport and no convection flow is given by $^{4}$ :

$$
j_{i}=-\frac{D_{k, i} c_{i}}{R T} \frac{\partial \mu_{i}}{\partial x}-\frac{z_{i} F}{R T} D_{k, i} c_{i} \frac{\partial \phi}{\partial x}
$$

where $\mu_{\mathrm{i}}[\mathrm{J}]$ denotes the chemical potential of the $i$ th species. To solve Eq. (6), let us consider the following ionic compound, which consists of $\mathrm{Li}^{+}$and $\mathrm{e}$.

If the neutral Li diffuses through the transition metal oxide under the electrochemical potential gradient in an open-circuit condition, the net current $I_{\text {net }}[\mathrm{A}]$ of the diffusion medium should be zero, i.e., $I_{\text {net }}=I_{\mathrm{Li}^{+}}$ $+I_{\mathrm{e}}=0$. Hence, the flux of $\mathrm{Li}^{+}$is matched correspondingly by an equivalent charge flux of $e$ in the same direction as shown in Fig. 2(a) as follows:

$$
j_{\mathrm{Li}^{+}}=j_{\mathrm{e}} \quad \text { (charge neutrality constraint) }
$$

By solving the flux density equation of Eq. (6) with the charge neutrality and stoichiometric constraints out of consideration of the local equilibrium constraint, the final flux equation of the neutral species $\mathrm{Li}$ is

$$
j_{L i}=-\left(\frac{2 D_{k, L i}{ }^{+} D_{k, e}}{D_{k, L i}+D_{k, e}}\right) \frac{\partial c_{L i}}{\partial x}=-\tilde{D_{L i}} \frac{\partial c_{L i}}{\partial x}
$$

Therefore, $\tilde{D}_{L i}$ is determined as follows:

$$
\tilde{D}_{L i}=\frac{2 D_{k, L i}{ }^{+} D_{k, e}}{D_{k, L i}{ }^{+}+D_{k, e}}
$$

On the other hand, to derive quantitatively the relation between the chemical potential gradient of $\mathrm{Li}^{+}\left(\partial \mu_{\mathrm{Li}^{+}} / \partial x\right)$ and the chemical potential gradient of neutral species $\mathrm{Li}\left(\partial \mu_{\mathrm{Li}} / \partial x\right)$, the local equilibrium constraint is frequently introduced by researchers to explain the real situation. If the electrochemical species is locally in the equilibrium with the diffusion medium during chemical diffusion, the general relationship of the local equilibrium constraint is given by: 


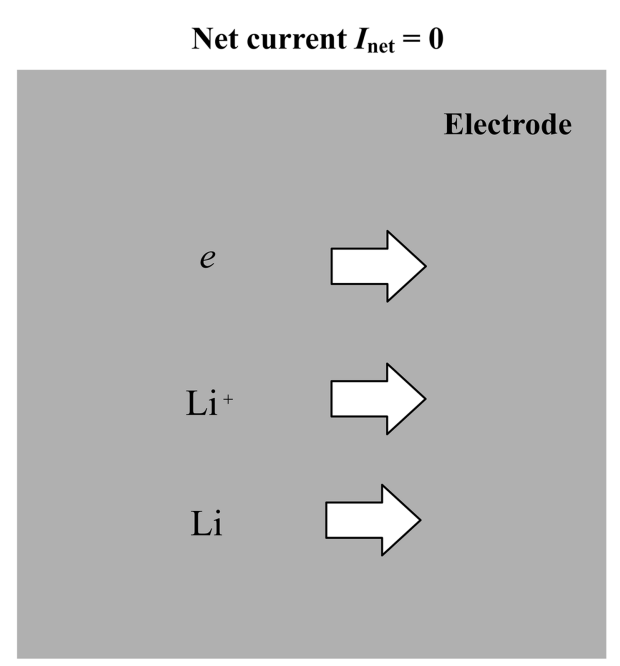

(a) Open-circuit condition

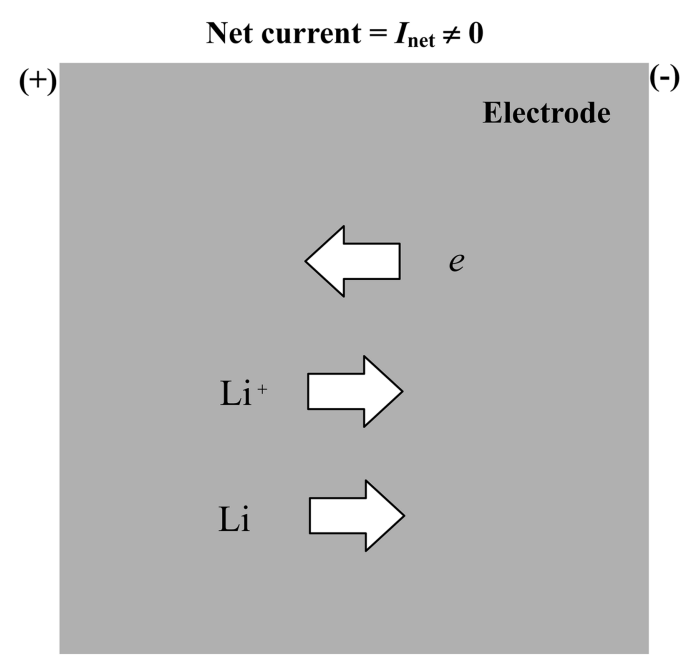

(b) Current flowing condition

Fig. 2. Schematic representations of chemical diffusion of Li through the transition metal oxide in (a) open circuit and (b) current flowing conditions.

$$
\eta_{\mathrm{Li}}=\eta_{\mathrm{Li}^{+}}+\eta_{\mathrm{e}}
$$

where $\eta_{\mathrm{i}}\left[\mathrm{J} \mathrm{mol}^{-1}\right]$ is the electrochemical potential of the $i$ th species, and the subscripts $\mathrm{Li}, \mathrm{Li}^{+}$and $e$ denote the neutral $\mathrm{Li}$ atom, $\mathrm{Li}^{+}$ion and electron, respectively.

By solving the flux density equation of Eq. (6) in consideration of the local equilibrium constraint of Eq. (10), the final flux equation of the neutral species Li is ${ }^{10,23)}$

$$
j_{L i}=\frac{D_{k, L i}{ }^{+} D_{k, e}}{D_{k, L i}+D_{k, e}} \frac{\partial \ln a_{L i}}{\partial \ln c_{L i}}\left(\frac{\partial c_{L i}}{\partial x}\right)
$$

Accordingly, we get

$$
\tilde{D}_{L i}=\frac{D_{k, L i}{ }^{+} D_{k, e}}{D_{k, L i}+D_{k, e}} \frac{\partial \ln a_{L i}}{\partial \ln c_{L i}}
$$

The detailed derivation of Eqs. (9) and (12) can be found elsewhere in the literature. ${ }^{23)}$

\subsection{Chemical diffusion in a current flowing condition}

Now let us derive the flux equation in a current flowing condition. Here, the current flowing condition means appreciably so small current flow under the negligibly small external electrical potential gradient. Comparing the Li transport in a current flowing condition with that in an open-circuit condition, it is suggested that $\mathrm{Li}^{+}$ions and electrons $e$ move in opposite directions in a current flowing condition as shown in Fig. 2(b). ${ }^{23)}$ From the general equation for the flux density of Eq. (6), the flux densities of $\mathrm{Li}^{+}$and e are given by:

$$
\begin{aligned}
& j_{L i^{+}}=-\left(D_{k, L i} \frac{\partial \ln a_{L i}}{\partial \ln c_{L i^{+}}} \frac{\partial c_{L i^{+}}}{\partial x}+\frac{F c_{L i^{+}} D_{k, L i}{ }^{+}}{R T} \frac{\partial \phi}{\partial x}\right) \\
& j_{e}=-\left(D_{k, e} \frac{\partial \ln a_{e} \partial c_{e}}{\partial \ln c_{e}} \frac{F c_{e} D_{k, e}}{\partial x} \frac{\partial \phi}{\partial x}\right)
\end{aligned}
$$

If the neutral $\mathrm{Li}$ atoms diffuse through the transition metal oxide under the electrochemical potential gradient in a current flowing condition, the flux balance between two different species is given as follows:

$$
t_{e}=\frac{I_{e}}{I_{n e t}}=\frac{I_{e}}{I_{L i^{+}}+I_{e}}=-\frac{F j_{e}}{F j_{L i^{+}}-F j_{e}}=-\frac{j_{e}}{j_{L i^{+}}-J_{e}}
$$

(charge neutrality constraint)

From Eqs. (13), (14) and (15), we get

$$
\begin{array}{r}
-t_{e}\left(D_{k, L i}{ }^{+} \frac{\partial c_{L i}{ }^{+}}{\partial x}+\frac{F c_{L i}{ }^{+} D_{k, L i^{+}}}{R T} \frac{\partial \phi}{\partial x}\right) \\
=\left(1-t_{e}\right)\left(D_{k, e} \frac{\partial c_{e}}{\partial x}-\frac{F C_{e} D_{k, e}}{R T} \frac{\partial \phi}{\partial x}\right)
\end{array}
$$

In addition to the charge neutrality constraint of Eq. (15), we consider the following stoichiometric constraint further

$$
\mathrm{c}_{\mathrm{Li}^{+}}=\mathrm{c}_{\mathrm{e}} \quad \text { (stoichiometric constraint) }
$$

By considering Eq. (17), Eq. (16) is rearranged into 


$$
\begin{aligned}
& t_{e} D_{k, L i}{ }^{+} \frac{\partial c_{L i} i^{+}}{\partial x}+\left(1-t_{e}\right) D_{k, e} \frac{\partial c_{e}}{\partial x} \\
& =-\frac{F c_{L i} D_{k, L i}{ }^{+} t_{e}}{R T} \frac{\partial \phi}{\partial x}+\left(1-t_{e}\right) \frac{F c_{e} D_{k, e}}{R T} \frac{\partial \phi}{\partial x} \\
& =-\frac{F c_{L i^{+}}}{R T}\left[t_{e} D_{k, L i^{+}}-\left(1-t_{e}\right) D_{e}\right] \frac{\partial \phi}{\partial x}
\end{aligned}
$$

Here, it should be stressed that to derive the final flux equation of the neutral species $\mathrm{Li}$ in a current flowing condition, the relation between $\partial c_{\mathrm{Li}^{+}} / \partial x$ and $\partial c_{e} / \partial x$ should be known. To derive this relation quantitatively, we assumed that the concentration of electrons is distributed in the Gaussian shape across the electrode as follows ${ }^{24,25)}$ :

$$
c_{e}(x)=c_{e}^{o} \exp \left[-A(L-x)^{2}\right]
$$

where $c_{e}^{o}\left[\mathrm{~mol} \mathrm{~cm}{ }^{-3}\right]$ is the maximum electron concentration in the electrode, $A[-]$ means the constant.

From the fact that in a current flowing condition, $\mathrm{Li}^{+}$ and e move in opposite directions, the concentration of $\mathrm{Li}^{+}$is correspondingly given as follows

$$
c_{\mathrm{Li}^{+}}(x)=c_{\mathrm{Li}^{+}}^{\mathrm{o}} \exp \left[-A x^{2}\right]
$$

Under this circumstance, the relation between $\partial c_{\mathrm{Li}^{+}} / \partial x$ and $\partial c_{\mathrm{e}} / \partial x$ is written by

$$
\frac{\partial c_{e}}{\partial x}=\frac{\partial c}{L i_{i}^{+}}+B
$$

where $B[-]$ denotes the constant which has no physical meaning. Figs. 3(a) and (b) show the resulting concentration gradients of $\mathrm{Li}^{+}$and $e$ simulated theoretically in open-circuit and current flowing conditions, respectively.

Considering Eq. (21), Eq. (18) is given as:

$$
\begin{gathered}
t_{e} D_{k, L i} \frac{\partial c_{L i}^{+}}{\partial x}+\left(1-t_{e}\right) D_{k, e}\left(\frac{\partial c_{L i} i^{+}}{\partial x}+B\right) \\
=-\frac{F c_{L i^{+}}}{R T}\left[t_{e} D_{k, L i^{+}}-\left(1-t_{e}\right) D_{k, e}\right] \frac{\partial \phi}{\partial x}
\end{gathered}
$$

Thus, the internal electrical field is expressed by:

$$
\begin{aligned}
& \frac{\partial \phi}{\partial x}=\left[-\frac{F c_{L i^{+}}}{R T}\left\{t_{e} D_{k, L i}-\left(1-t_{e}\right) D_{k, e}\right\}\right]^{-1} \\
& \times\left[t_{e} D_{k, L i} \frac{\partial c^{+}}{\partial x}+\left(1-t_{e}\right) D_{k, e}\left(\frac{\partial c}{\partial i^{+}}+B\right)\right]
\end{aligned}
$$

Inserting Eq. (23) into Eq. (13), the flux equation yields

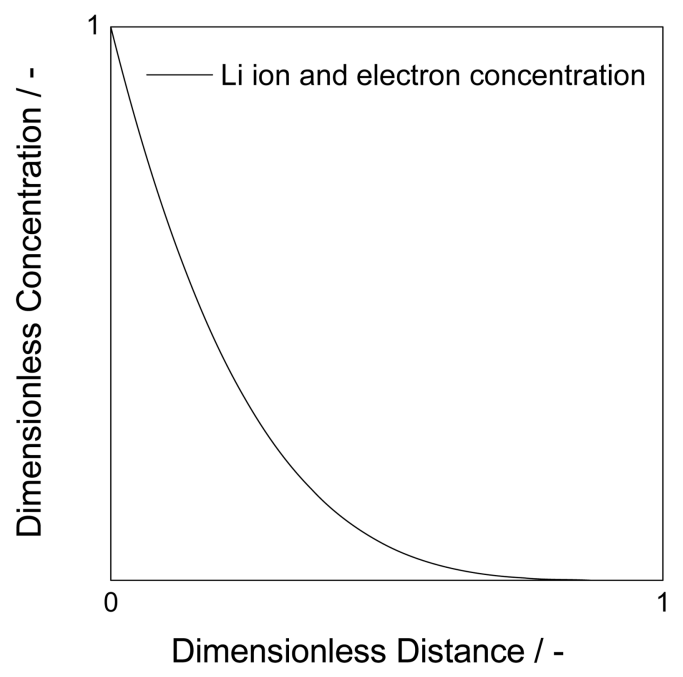

(a) Open-circuit condition

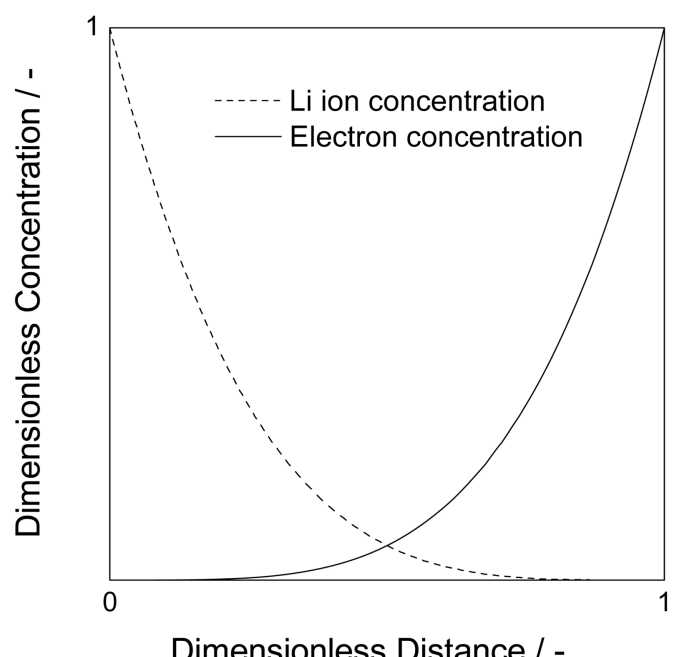

(b) Current flowing condition

Fig. 3. Dimensionless concentration profiles of $\mathrm{Li}^{+}$and e in the electrode theoretically calculated in (a) open circuit and (b) current flowing conditions.

$$
\begin{aligned}
& j_{L i^{+}}=\left[-D_{k, L i}{ }^{{ }^{+}} \frac{\partial c}{\partial x}-\left(\frac{D_{k, L i}{ }^{+}}{t_{e} D_{k, L i} i^{-}\left(1-t_{e}\right) D_{k, e}}\right)\right. \\
& \left.\times\left\{t_{e} D_{k, L i}{ }^{+} \frac{\partial c}{\partial x}+\left(1-t_{e}\right) D_{k, e}\left(\frac{\partial c_{L i} i^{+}}{\partial x}+B\right)\right\}\right]
\end{aligned}
$$


In order to obtain the chemical diffusivity of the neutral $\mathrm{Li}$, it is first assumed that both $\mathrm{Li}^{+}$and $\mathrm{e}$ show an ideal solution behaviour. From this assumption and Eq. (24), the chemical potential gradient can be expressed as follows:

$$
\begin{aligned}
& \frac{\partial \mu_{L i^{+}}}{\partial x}=R T \frac{\partial \ln a_{L i^{+}}}{\partial x}=\frac{R T}{c_{L i}} \frac{\partial c_{L i}^{+}}{\partial x} \\
& \frac{\partial \mu_{e}}{\partial x}=R T \frac{\partial \ln a_{e}}{\partial x}=\frac{R T}{c_{e}} \frac{\partial c_{e}}{\partial x}
\end{aligned}
$$

In addition, by assuming that chemical diffusion through the stoichiometric ionic compound may occur via a vacancy mechanism satisfying the charge neutrality condition, the flux and concentration of each species satisfies the following relationship.

$$
\begin{gathered}
j_{\mathrm{Li}}=j_{\mathrm{Li}^{+}}=j_{e} \\
c_{\mathrm{Li}}=c_{\mathrm{Li}^{+}}=c_{e}
\end{gathered}
$$

By considering Eqs. (25), (26), (27) and (28), the final flux equation of the neutral $\mathrm{Li}$ is given by

$$
\begin{aligned}
& j_{L i^{+}}=-\left[-\frac{2\left(1-t_{e}\right) D_{k, L i}{ }^{+} D_{k, e}}{t_{e} D_{k, L i^{+}}-\left(1-t_{e}\right) D_{k, e}}\right] \frac{\partial c_{L i^{+}}}{\partial x} \\
& +\left[\frac{\left(1-t_{e}\right) D_{k, L i}{ }^{+} D_{k, e}}{t_{e} D_{k, L i^{+}}-\left(1-t_{e}\right) D_{k, e}}\right] B
\end{aligned}
$$

Therefore, the chemical diffusivity of the neutral $\mathrm{Li}$ atoms $\tilde{D}_{L i}$ is determined as follows:

$$
\tilde{D}_{L i}=-\frac{2\left(1-t_{e}\right) D_{k, L i}{ }^{+} D_{k, e}}{t_{e} D_{k, L i}{ }^{+}-\left(1-t_{e}\right) D_{k, e}}
$$

Similar to the previous section, in case that the electrochemical species is locally in the equilibrium with the diffusion medium during chemical diffusion, the final flux equation of the neutral species $\mathrm{Li}$ in consideration of the local equilibrium is

$$
j_{L i^{+}}=-\left[-\frac{\left(1-t_{e}\right) D_{k, L i}{ }^{+} D_{k, e}}{t_{e} D_{k, L i}{ }^{+}\left(1-t_{e}\right) D_{k, e}} \frac{d \ln a_{L i}}{d \ln c_{L i}}\right] \frac{\partial c_{L i}{ }^{+}}{\partial x}
$$

Accordingly, we get

$$
\tilde{D}_{L i}=-\frac{\left(1-t_{e}\right) D_{k, L i}{ }^{+} D_{k, e}}{t_{e} D_{k, L i}{ }^{+}-\left(1-t_{e}\right) D_{k, e}} \frac{d \ln a_{L i}}{d \ln c_{L i}}
$$

\subsection{Comparative discussion on chemical diffusion} in two different conditions

In the previous two sections, the four different equations, Eqs. (9), (12), (30) and (32), for the chemical diffusivities were derived under four different constraints. From the fact that the component diffusivity of electrons is much higher than that of Li ions, the resulting chemical diffusivities of the neutral Li can be simplified as in an open-circuit condition

$\tilde{D}_{L i}=2 D_{k, L i^{+}} \quad$ (without the local equilibrium)(33)

$$
\tilde{D}_{L i}=D_{k, L i} \frac{\partial \ln a_{L i}}{\partial \ln c_{L i}}
$$

(with the local equilibrium)

in a current flowing condition

$$
\begin{aligned}
\tilde{D}_{L i} & =2 D_{k, L i^{+}} \quad(\text { without the local equilibrium)(35) } \\
\tilde{D}_{L i} & =D_{k, L i} \frac{\partial \ln a_{L i}}{\partial \ln c_{L i}}
\end{aligned}
$$

(with the local equilibrium)

Either Eqs. (33) and (35) or Eqs. (34) and (36) imply that in the transition metal oxide, the chemical diffusivity in an open-circuit condition is exactly the same as that in a current flowing condition. In both conditions, it concerns whether or not the local equilibrium constraint is considered.

Here, it would be very interesting to compare these two cases, in consideration and out of consideration of the local equilibrium constraints. The discordance of the two equations might originate from the applicability of the local equilibrium constraint throughout the transition metal oxide. For the derivation of Eq. (33), it is assumed that the chemical potential of the neutral $\mathrm{Li}$ is the sum of the two chemical potentials of the charged species $\mathrm{Li}^{+}$and $\mathrm{e}$. This assumption proves to be always proper at the entry and exit sides, but it is still open to discussion whether Eq. (34) is valid or not within the diffusion medium.

This means that if the electroactive species might be activated locally during chemical diffusion through the diffusion medium, the local equilibrium constraint is no longer valid within the diffusion medium. In this case, the electroactive species is not thermodynamically in equilibrium with the diffusion medium and hence the expression of chemical diffusivity Eq. (34) is no longer effective. From this argument, it is suggested that the expression of chemical diffusivity is not uniquely 
defined, but it depends upon the thermodynamic or electrochemical condition of the diffusion media. Nevertheless, the origin of the local equilibrium constraint still needs to be clarified.

\section{Electrochemical methods to determine experimentally the chemical diffusion coefficient}

Several electrochemical methods have been developed to determine the chemical diffusivity of the neutral species $\mathrm{Li}$ in the transition metal oxide. The methods are classified into a transient technique and a steady-state technique. In the quasi-steady-state technique, the impedance oscillation is obtained from the relationship between the potential oscillation applied to the electrode and the resulting current oscillation. In the transient technique, on the other hand, which indicates the experimental condition whereby Li diffuses in a current flowing condition, just after application of either the current pulse or potential pulse to the electrode, the resulting potential transient or current transient is measured, respectively.

\subsection{Quasi-steady-state method - Ac impedance spectroscopy}

To evaluate the chemical diffusivity, one fits the measured impedance spectra to an equivalent circuit representing the physical processes taking place in the system under investigation. At a thermodynamically stable single phase region, the Warburg impedance in complex number $\underline{Z}_{\mathrm{w}}$ representing the Li diffusion in the electrode can be expressed as

$$
\underline{Z}_{W}=\frac{C}{\sqrt{j \omega}}=\frac{C}{\sqrt{2}} \omega^{-1 / 2}(1-j)=Z^{\prime}-j Z^{\prime \prime}
$$

where $C[-]$ is the constant, $j[-]$ the unit of imaginary part of the complex number, $\omega[\mathrm{Hz}]$ the angular frequency, and $Z^{\prime}[\Omega]$ and $Z^{\prime \prime}[\Omega]$ represent the real and imaginary parts of $\underline{Z}_{\mathrm{W}}$. The Warburg coefficient $\sigma_{\mathrm{W}}$ can be expressed as

$$
\sigma_{W}=\frac{C}{\sqrt{2}}=\left[\frac{V_{m}}{F}\left(\frac{d E}{d \delta}\right) \frac{1}{\sqrt{2 \tilde{D}_{L i}}}\right] \frac{1}{A_{e a}}
$$

where $V_{\mathrm{m}}\left[\mathrm{cm}^{3} \mathrm{~mol}^{-1}\right]$ is the molar volume of the electrode material, $E[\mathrm{~V}]$ the electrode potential, $\delta[-]$ the deviation from the ideal stoichiometry, and $A_{\text {ea }}\left[\mathrm{cm}^{2}\right]$ denotes the electrochemical active area of the electrode. In our previous works, ${ }^{26-29)}$ the chemical diffusivity of Li $\tilde{D}_{L i}$ in the various transition metal oxides was determined from the measured impedance spectra by using Eq. (38). The $\tilde{D}_{L i}$ values were estimated to $10^{-10}$ to $10^{-12} \mathrm{~cm}^{2} \mathrm{~s}^{-1}$ for the $\mathrm{LiV}_{2} \mathrm{O}_{5}$ electrode, ${ }^{28)} 10^{-7}$ to $10^{-9} \mathrm{~cm}^{2} \mathrm{~s}^{-1}$ for the $\mathrm{LiNiO}_{2}$ electrode, ${ }^{26} 10^{-9}$ to $10^{-10} \mathrm{~cm}^{2} \mathrm{~s}^{-1}$ for the $\mathrm{LiCoO}_{2}$ electrode, ${ }^{29)}$ and $10^{-8} \mathrm{~cm}^{2} \mathrm{~s}^{-1}$ for the $\mathrm{LiMn}_{2} \mathrm{O}_{4}$ electrode. ${ }^{27)}$

\subsection{Transient method - Potentiostatic and} galvanostatic methods

3.2.1. Galvanostatic intermittent titration technique (GITT)

This method involves the application of a constant current through the electrode for a time interval. Then, the potential difference is measured between the working electrode and the reference electrode as a function of time during the current application. In this method, the chemical diffusivity of Li into a mixed conductor electrode in planar symmetry can be calculated by using the following equation as a function of Li content

$$
\begin{aligned}
& \tilde{D}_{L i}=\frac{4}{\pi}\left(\frac{V_{m}}{z F A_{e a}}\right)^{2}\left[\frac{I_{a p p}\left(\frac{d E}{d \delta}\right)}{\left(\frac{d E}{d \sqrt{t}}\right)}\right]^{2} \\
& \text { for } \mathrm{t}<<\frac{L^{2}}{\tilde{D}_{L i}} \quad \text { (semi-infinite condition) }
\end{aligned}
$$

where $I_{\text {app }}[\mathrm{A}]$ and $t[\mathrm{~s}]$ mean the applied constant current and time, respectively.

Since the GITT method places no limits on the initial and boundary conditions for Fick's second law, and hence gives a linear relationship between the potential and the square root of time in the short time range of the galvanostatic potential transient, it seems appropriate to determine Li diffusivity in a mixed conductor electrode.

In our previous works, ${ }^{30-33)}$ the chemical diffusivity of Li $\tilde{D}_{L i}$ in the different transition metal oxides was evaluated by analysis of the measured GITT using Eq. (39). The $\tilde{D}_{L i}$ values were determined to $10^{-11}$ to $10^{-13} \mathrm{~cm}^{2} \mathrm{~s}^{-1}$ for the $\mathrm{LiV}_{2} \mathrm{O}_{5}$ electrode, ${ }^{30)} 10^{-8}$ to $10^{-9} \mathrm{~cm}^{2} \mathrm{~s}^{-1}$ for the $\mathrm{LiNiO}_{2}$ electrode, ${ }^{31)} 10^{-8}$ to $10^{-9} \mathrm{~cm}^{2} \mathrm{~s}^{-1}$ for the $\mathrm{LiCoO}_{2}$ electrode, ${ }^{32)}$ and $10^{-7}$ to $10^{-8} \mathrm{~cm}^{2} \mathrm{~s}^{-1}$ for the $\mathrm{LiMn}_{2} \mathrm{O}_{4}$ electrode. ${ }^{33)}$

\subsubsection{Potentiostatic intermittent titration} technique (PITT)

This technique involves the application of a sudden step in the potential across the electrode. In such 
experiments, it is assumed that a planar electrode initially has a uniform concentration of the mobile electroactive species, i.e., Li, corresponding to an equilibrium potential with respect to the reference electrode. At $t=0$, a new activity, which is proportional to the concentration of $\mathrm{Li}$, is imposed on the electrode surface by applying a potential step between the sample and the reference electrode. Chemical diffusion will occur due to the concentration gradient imposed within the electrode. As a result, the Li must be supplied continuously by transport through the electrolytic phase in order to keep the surface concentration constant, until the electrode reaches the equilibrium composition everywhere.

An advantage of using the PITT method is that side reactions such as the nucleation of new phases can be avoided if the potentials are controlled within the stability range of the single phase. However, a disadvantage is that the ohmic potential drop in the bulk electrolyte, which varies with time, can not be eliminated readily from the applied potential difference $\Delta E$. By solving Fick's second law, the chemical diffusivity of Li in the electrode can be expressed as

$$
\begin{aligned}
& \tilde{D}_{L i}=\frac{\pi}{z F A_{e a} c^{s}}\left(\frac{d I(t)}{d 1 / \sqrt{t}}\right)^{2} \\
& \text { for } \mathrm{t}<<\frac{L^{2}}{\tilde{D}_{L i}} \quad \text { (semi-infinite condition) }
\end{aligned}
$$

where $c^{\mathrm{s}}\left[\mathrm{mol} \mathrm{cm}^{-3}\right]$ is the Li concentration at the electrode/electrolyte interface and $I(t)[\mathrm{A}]$ represents the current flowing at the electrode/electrolyte interface.

In our previous works, ${ }^{34,35)}$ the chemical diffusivity of $\mathrm{Li} \tilde{D}_{L i}$ in the different transition metal oxides was calculated by analysing the measured PITT employing Eq. (40). The $\tilde{D}_{L i}$ values were determined to $10^{-12}$ to $10^{-13} \mathrm{~cm}^{2} \mathrm{~s}^{-1}$ for the $\mathrm{LiV}_{2} \mathrm{O}_{5}$ electrode ${ }^{34)}$ and $10^{-8}$ to $10^{-9} \mathrm{~cm}^{2} \mathrm{~s}^{-1}$ for the $\mathrm{LiCoO}_{2}$ electrode. ${ }^{35)}$ The resulting
$\tilde{D}_{L i}$ values are listed in Table 1. From Table 1 , it is concluded that the $\tilde{D}_{L i}$ value experimentally determined in an open-circuit condition is almost the same as that in a current flowing condition, which is matched well with the theoretical results presented in Section 2.

\section{Discussion}

In this work, it is necessary to discuss the thermodynamic enhancement factor $W_{\text {th }}$ in more detail. The $W_{\text {th }}$ is generally defined as

$$
W_{t h}=\frac{\partial \ln a_{L i}}{\partial \ln c_{L i}}
$$

The $W_{\text {th }}$ value is only capable of enhancing the motion of the ions. In the case of very low diffusivities, $W_{\text {th }}$ may enhance chemical diffusion by many orders of magnitude, but the chemical diffusion coefficient may still be low. In contrast, one might think that a high diffusivity would not require any further enhancement. Based upon our present state of knowledge, the ionic diffusivities in metals and semiconductors are rather similar and it is therefore helpful to make use of large $W_{\text {th }}$ values. This is especially helpful because there are ways to control this factor by suitable doping of semiconductors. ${ }^{5 \text { ) }}$

In order to produce a large $W_{\text {th }}$ value, the concentration of the mobile ions should be high compared to the concentration of the electronic species. However, the ionic concentration $c_{i}$ should not be very much larger than the electron concentration $c_{e}$ in order to keep the transference number of the electrons close to 1 . These requirements are somewhat contradictory, but may best be fulfilled if the mobility of the small number of electrons is very large compared to the mobility of the ions. In this way, the conductivity of the electrons

Table 1. The chemical diffusivity of $\mathrm{Li} \tilde{D}_{L i}$ determined by using ac impedance spectroscopy, galvanostatic intermittent titration technique (GITT) and potentiostatic intermittent titration technique (PITT)

\begin{tabular}{lccc} 
& \multicolumn{3}{c}{ Chemical diffusivity of $\mathrm{Li}\left[\mathrm{cm}^{2} \mathrm{~s}^{-1}\right]$} \\
\cline { 2 - 4 } Specimen & $\begin{array}{c}\text { Transient method } \\
\text { Ac impedance spectroscopy }\end{array}$ & $\begin{array}{c}\text { Galvanostatic intermittent titration } \\
\text { technique (GITT) }\end{array}$ & $\begin{array}{c}\text { Potentiostatic intermittent titration } \\
\text { technique (PITT) }\end{array}$ \\
\cline { 3 - 4 } $\mathrm{LiV}_{2} \mathrm{O}_{5}$ & $10^{-10}-10^{-1228)}$ & $10^{-11}-10^{-1330)}$ & $10^{-12}-10^{-1334)}$ \\
$\mathrm{LiNiO}_{2}$ & $10^{-7}-10^{-926)}$ & $10^{-8}-10^{-931)}$ & - \\
$\mathrm{LiCoO}_{2}$ & $10^{-9}-10^{-1029)}$ & $10^{-8}-10^{-932)}$ & $10^{-8}-10^{-935)}$ \\
$\mathrm{LiMn}_{2} \mathrm{O}_{4}$ & $\left.10^{-8} 27\right)$ & $10^{-7}-10^{-833)}$ & - \\
\hline
\end{tabular}


may be kept larger than the conductivity of the ions. In order to come up with a quantitative relationship, the transference number of the electrons is substituted by the product of the concentrations and the diffusivities or mobilities.

The large enhancement factors higher than 10 are obtained at sufficiently low electronic concentrations and high electronic mobilities. This situation is most favourable for semiconductors that commonly fulfill both requirements, since the mobility of the electrons is commonly much larger in semiconducting material than in metallic material. In this case, the faster electrons accelerate the motion of the slower $\mathrm{Li}^{+}$ions and at the same time, the diffusion of electrons is retarded by the influence of the slower $\mathrm{Li}^{+}$ions. As the electron concentration increases, the interaction between electrons and $\mathrm{Li}^{+}$ions decreases, and hence the value of $W_{\text {th }}$ approaches to 1 .

In our previous works, ${ }^{30,33,36)}$ the $W_{\text {th }}$ value was estimated from the following relation

$$
\begin{aligned}
& \tilde{D}_{L i}=D_{k, L i} \frac{\partial \ln a_{L i}}{\partial \ln c_{L i}}=D_{k, L i} W_{t h} \\
& \text { with } W_{t h}=-\frac{z F \delta}{R T}\left(\frac{d E}{d \delta}\right)
\end{aligned}
$$

The $W_{\text {th }}$ values were determined experimentally by analysis of the measured GITT curves using Eq. (42). The resulting $W_{\text {th }}$ values ranged from 6 to 15 for the $\mathrm{LiV}_{2} \mathrm{O}_{5}$ electrode, ${ }^{30)}$ from 50 to 1000 for the $\mathrm{LiNiO}_{2}$ electrode, ${ }^{36)}$ and from 2 to 100 for the $\mathrm{LiMn}_{2} \mathrm{O}_{4}$ electrode, ${ }^{33)}$ which are listed in Table 2.

In contrast to $W_{\mathrm{th}} \geq 1$, if the mobility of the electrons is low, the values of $W_{\text {th }}<1$ may be observed. This condition is only required for such solid electrolytes as stabilised $\mathrm{ZrO}_{2}\left(\mathrm{t}_{\mathrm{O}^{2-}} \leq 1\right)$ in which the motion of the ions is blocked because no charge compensating electrons are available. The requirement for electrolytes is very low electronic mobility rather than a low concentration of electrons. In this case, the faster oxygen

Table 2. The thermodynamic enhancement factor $W_{\text {th }}$ determined experimentally by analysis of the measured galvanostatic intermittent titration curves using Eq. (42).

\begin{tabular}{lc}
\hline Specimen & Thermodynamic enhancement factor $W_{\text {th }}[-]$ \\
\hline $\mathrm{LiV}_{2} \mathrm{O}_{5}$ & $6-15[30]$ \\
$\mathrm{LiNiO}_{2}$ & $50-1000[36]$ \\
$\mathrm{LiCoO}_{2}$ & - \\
$\mathrm{LiMn}_{2} \mathrm{O}_{4}$ & $2-100[33]$ \\
\hline
\end{tabular}

ions $\mathrm{O}^{2-}$ accelerate the motion of the slower electrons and at the same time, the $\mathrm{O}^{2-}$ diffusion is decelerated by the slower electrons. Under this condition, chemical diffusion of $\mathrm{O}^{2-}$ ions occurs hardly, but $\mathrm{O}^{2-}$ ions transport proceeds by purely migration rather than diffusion under the external electrical potential gradient.

From the point of view of these electronic properties, electrodes and electrolytes are clearly distinguishable from each other. Electrodes should have highly mobile electronic species whereas electrolytes require low electronic mobilities. Studies of the electronic properties are therefore very useful in the search for new electrode and electrolyte materials.

\section{Acknowledgements}

This study was supported by a grant from the Center for Advanced Materials Processing (CAMP) of the 21st Century Frontier R\&D Program funded by the Ministry of Commerce, Industry and Energy (MOCIE), Republic of Korea.

\section{References}

1. L. Onsager and S. Machlup, "Fluctuations and Irreversible Processes", Phys. Rev., 91, 1505 (1953).

2. S. Machlup and L. Onsager, "Fluctuations and Irreversible Processes. II. Systems with Kinetic Energy", Phys. Rev., 91, 1512 (1953).

3. T. Z. Fahidy and S. Mohanta, "Mass transport in electrochemical systems" in Advances in transport processes, 84-92, ed. A.S. Mujumdar and R.A. Mashelkar, John Wiley \& Sons, New York (1980).

4. A. J. Bard and L. R. Faulkner, "Electrochemical methods", 28-35, John Wiley \& Sons, New York (1980).

5. W. Weppner, "Electrode Performance", in Solid State Electrochemistry, 199-228, ed. P.G. Bruce, Cambridge Univ. Press, Cambridge (1995).

6. S. -I. Pyun, "What Do We Understand by Chemical and Component Diffusivities of Cation in Mixed Conductor? - Basic Concepts and Electrochemical Techniques to Their Determination at Cathode Materials of Secondary Lithium Battery", J. Corr. Sci. of Korea, 28, 1 (1999).

7. F. A. Kroeger, "The Chemistry of Imperfect Crystals", 794-813, North-Holland Publishing Company, Amsterdam (1964).

8. S. R. De Groot and P. Mazur, "Non-Equilibrium Thermodynamics", 238-273, North-Holland Publishing Company, Amsterdam (1969).

9. H. Schmalzried, "Solid State Reactions", in Monographs in Modern Chemistry, 59-91, ed. H.F. Ebel, Verlag Chemie, Weinheim (1981). 
10. H. Schmalzried, "Chemical Kinetics of Solids", 95-107, VCH Verlagsgesellschaft mbH, Weinheim (1995).

11. W. Van. Gool, "Principles of defect chemistry of crystalline solids", 13-25, Academic Press, New York (1966).

12. G. Balducci, J. Kaspar, P. Fornasiero, M. Graziani, M. S. Islam, and J. D. Gale, "Computer simulation studies of bulk reduction and oxygen migration in $\mathrm{CeO}_{2}-\mathrm{ZrO}_{2}$ solid solutions", J. Phys. Chem. B, 101, 1705 (1997).

13. M. Kilo, R. A. Jackson, and G. Borchardt, "Computer modelling of ion migration in zirconia", Phil. Mag., 83, 3309 (2003).

14. P. -W. Li, L. Schaefer, and M. K. Chyu, "Multiple transport processes in solid oxide fuel cells" in Transport phenomena in fuel cells, 1-42, ed. B. Sunden and M. Faghri, WIT Press, Boston (2005).

15. J. -S. Kim, S. -I. Pyun, J. -W. Lee, and R. -H. Song, "Kinetics of oxygen reduction on porous mixed conducting $\left(\mathrm{La}_{0.85} \mathrm{Sr}_{0.15}\right)_{0.9} \mathrm{MnO}_{3}$ electrode by ac-impedance analysis", J. Solid State Electrochem., 11, 117 (2007).

16. J. -S. Kim, and S. -I. Pyun, "Effects of secondary phase and thickness on mixed controlled oxygen reduction at dense composite electrode", J. Electrochem. Soc., 155, B8 (2008).

17. J. -S. Kim, S. -I. Pyun, H. -C. Shin, and S. -J. L. Kang, "Oxygen reduction kinetics at dense $\left(\mathrm{La}_{0.85} \mathrm{Sr}_{0.15}\right)_{0.9} \mathrm{MnO}_{3}$ -YSZ composite electrodes investigated using potentiostatic current transient method", J. Electrochem. Soc., 155, B762 (2008).

18. J. -S. Kim and S. -I. Pyun, "Kinetics of oxygen reduction at composite electrodes with controlled three-phase boundaries by patterning YSZ column", Electrochim. Acta, 54, 952 (2009).

19. J. -S. Kim, S. -I. Pyun, and H. -C. Shin, "Kinetics of cathodic reaction at porous composite electrode under mixed control of migration and oxygen-exchange", $J$. Electrochem. Soc., submitted for publication (2009).

20. A. Kumar, D. Rajdev, and D.L. Douglass, "Effect of oxide defect structure on the electrical properties of $\mathrm{ZrO}_{2}$ ", J. Amer. Ceram. Soc., 55, 439 (1972).

21. S. -I. Pyun and Y. -E. Ihm, "Electrical conduction of partially stabilized zirconia $\mathrm{Zr}_{0.94} \mathrm{Ca}_{0.06} \mathrm{O}_{1.94}$ as a function of temperature and oxygen partial pressure", J. Mat. Sci., 17, 2577 (1982).

22. R. J. Chater, S. Carter, J. A. Kilner, and B. C. H. Steele, "Development of a novel SIMS technique for oxygen self-diffusion and surface exchange coefficient measurements in oxides of high diffusivity", Solid State Ionics, 53-56, 859 (1992).

23. S. -J. Lee, S. -I. Pyun, and H. -C. Shin, "Chemical diffusion of electroactive species in ionic compounds: a focus on chemical equilibrium constraint", J. Solid State
Electrochem., in press (2009).

24. T. -W. Tang, T. O. Regan, and B. Wu, "Thomas-Fermi approximation for a two-dimensional electron gas at low temperatures", J. Appl. Phys., 95, 7990 (2004).

25. D. N. Quang, L. Tuan, and N.T. Tien, "Electron mobility in Gaussian heavily doped $\mathrm{ZnO}$ surface quantum wells", Phys. Rev. B, 77, 125326 (2008).

26. Y.-M. Choi, S.-I. Pyun and S.-I. Moon, "Effects of cation mixing on the electrochemical lithium intercalation reaction into porous $\mathrm{Li}_{1-\mathrm{d}} \mathrm{Ni}_{1-\mathrm{y}} \mathrm{Co}_{\mathrm{y}} \mathrm{O}_{2}$ electrodes", Solid State Ionics, 89, 43 (1996).

27. S. -I. Pyun, Y. -M. Choi, and I. -D. Jeng, "Effect of the lithium content on electrochemical lithium intercalation into amorphous and crystalline powdered $\mathrm{Li}_{1+\delta} \mathrm{Mn}_{2} \mathrm{O}_{4}$ electrodes prepared by sol-gel method", J. Power Sources, 68, 593 (1997).

28. S. -I. Pyun and J. -S. Bae, "Electrochemical lithium intercalation into vanadium pentoxide xerogel film electrode", J. Power Sources, 68, 669 (1997).

29. H. -C. Shin and S. -I. Pyun, "Investigation of lithium transport through lithium cobalt dioxide thin film sputter-deposited by analysis of cyclic voltammogram", Electrochim. Acta, 46, 2477 (2001).

30. J. -S. Bae and S. -I. Pyun, "Electrochemical lithium intercalation reaction of anodic vanadium oxide film", $J$. Alloys \& Comp., 217, 52 (1995).

31. Y. -M. Choi, S. -I. Pyun, J. -S. Bae, and S. -I. Moon, "Effects of lithium content on the electrochemical lithium intercalation reaction into $\mathrm{LiNiO}_{2}$ and $\mathrm{LiCoO}_{2}$ electrodes", J. Power Sources, 56, 25 (1995).

32. Y. -M. Choi and S. -I. Pyun, "Determination of electrochemical active area of porous $\mathrm{Li}_{1-\delta} \mathrm{CoO}_{2}$ electrode using the GITT technique", Solid State Ionics, 109, 159 (1998).

33. S. -W. Kim and S. -I. Pyun, "Thermodynamic and kinetic approaches to lithium intercalation into a $\mathrm{Li}_{1-\delta} \mathrm{Mn}_{2} \mathrm{O}_{4}$ electrode using Monte Carlo simulation", Electrochim. Acta, 46, 987 (2001).

34. J. -S. Bae and S. -I. Pyun, "Electrochemical lithium intercalation into and deintercalation from vanadium oxide electrode by using potentiostatic current transient technique", Solid State Ionics, 90, 251 (1996).

35. S. -I. Pyun and Y. -M. Choi, "Electrochemical lithium intercalation into and de-intercalation from porous $\mathrm{LiCoO}_{2}$ electrode by using potentiostatic current transient technique", J. Power Sources, 68, 524 (1997).

36. Y. -M. Choi, S. -I. Pyun, S. -I. Moon, and Y. -E. Hyung, "A study of the electrochemical lithium intercalation behavior of porous $\mathrm{LiNiO}_{2}$ electrodes by solid-state reaction and sol-gel methods", J. Power Sources, 72, 83 (1998). 